\title{
Uncertainty relation between angle and orbital angular momentum: interference effect in electron vortex beams
}

\author{
Shogo Tanimura
}

Department of Complex Systems Science, Graduate School of Information Science, Nagoya University, Nagoya 464-8601, Japan

tanimura@is.nagoya-u.ac.jp

\section{PACS 03.65.-w, 03.65.Ta, 07.78.+s, 42.50.-p, 42.50.Tx DOI 10.17586/2220-8054-2015-6-2-205-212}

The uncertainty relation between angle and orbital angular momentum had not been formulated in a similar form as the uncertainty relation between position and linear momentum because the angle variable is not represented by a quantum mechanical self-adjoint operator. Instead of the angle variable operator, we introduce the complex position operator $\hat{Z}=\hat{x}+i \hat{y}$ and interpret the order parameter $\mu=\langle\hat{Z}\rangle / \sqrt{\langle\hat{Z} \dagger \hat{Z}\rangle}$ as a measure of certainty of the angle distribution. We prove the relation between the uncertainty of angular momentum and the angle order parameter. We also prove its generalizations and discuss experimental methods for testing these relations.

Keywords: uncertainty relation, orbital angular momentum, order parameter, vortex electron beam.

Received: 4 November 2014

\section{Introduction}

Uncertainty relations elucidate the difference between classical physics and quantum physics. In classical physics, accuracy of measurement is not limited in principle and it is assumed that any observables can be measured simultaneously and precisely. However, in quantum physics, the accuracy of simultaneous measurements of two observables is limited by the uncertainty relation.

Originally, Heisenberg [1] formulated the uncertainty relation between position $Q$ and linear momentum $P$ as:

$$
\Delta Q \Delta P \gtrsim h
$$

with the Planck constant $h$. He deduced this relation via a Gedankenexperiment. Later, Weyl, Kennard, and Robertson [2] gave a rigorous proof of this relation. In the context of quantum mechanics, the position is represented by a self-adjoint operator $\hat{Q}$ and the uncertainty of the position is defined as the variance:

$$
(\Delta Q)^{2}:=\left\langle\psi\left|(\hat{Q}-\langle\psi|\hat{Q}| \psi\rangle)^{2}\right| \psi\right\rangle=\left\langle\psi\left|\hat{Q}^{2}\right| \psi\right\rangle-\langle\psi|\hat{Q}| \psi\rangle^{2},
$$

for a normalized state vector $|\psi\rangle$. The uncertainty $\Delta P$ of momentum is defined in a similar way.

It is natural to expect a similar relation:

$$
\Delta \phi \Delta L \gtrsim h,
$$

holds for the angle $\phi$ and the angular momentum $L$ as shown in the textbook [3]. However, in a plane, the coordinate values $\{\phi+2 \pi n\}$ with any integer $n$ represent the same point as $\phi$ indicates. In other words, the angle variable $\phi$ is a multivalued function. In quantum 
mechanics, the spectrum of a self-adjoint operator should have one-to-one correspondence with the values of an observable. Hence, there is no self-adjoint operator $\hat{\phi}$ corresponding to the multivalued angle variable $\phi$. Therefore, the angle uncertainty $\Delta \phi$ cannot be defined as the position uncertainty $\Delta Q$ was defined.

The uncertainty relation between angle and orbital angular momentum is a longstanding issue in physics. Many people have proposed other definitions of the angle uncertainty and have formulated several versions of the uncertainty relation between angle and angular momentum [4] - [9]. However, most of these relations treat a particle moving on a one-dimensional circle. They did not consider a particle moving in two- or threedimensional spaces. Thus, we do not yet have an angle-angular momentum uncertainty relation that is applicable for a realistic situation.

In this paper, we introduce the moment of position distribution in a plane, which is an arbitrary two-dimensional subspace in the configuration space of the particle. We propose to use the moment of position as an indicator of certainty or bias of angle distribution. The main results of this work are inequalities (27), (32), (36), which represent the uncertainty relation between the moments of position and the orbital angular momentum. Our results are applicable for a particle moving in configuration space having more than two dimensions.

\section{Robertson inequality}

The Robertson inequality [2] is one formulation of general uncertainty relations. The Robertson inequality has a clear meaning and it is applicable to any kind of observable. Hence, it is regarded as the universal formulation of uncertainty relations. Although the Robertson inequality is well known and its proof is rather simple, here, we write its derivation to make a comparison with our uncertainty relation of the angle and angular momentum, which is derived in the next section.

For any vectors $|\alpha\rangle$ and $|\beta\rangle$ of a Hilbert space $\mathscr{H}$, the Schwarz inequality:

$$
\langle\alpha \mid \alpha\rangle\langle\beta \mid \beta\rangle \geq|\langle\alpha \mid \beta\rangle|^{2}
$$

holds. The equality holds if and only if the two vectors $|\alpha\rangle$ and $|\beta\rangle$ are linearly dependent. Let $\psi \in \mathscr{H}$ be an arbitrary normalized vector satisfying $\langle\psi \mid \psi\rangle=1$. For self-adjoint operators $\hat{A}$ and $\hat{B}$ on $\mathscr{H}$, we set:

$$
\begin{aligned}
& \langle\hat{A}\rangle:=\langle\psi|\hat{A}| \psi\rangle, \\
& \Delta \hat{A}:=\hat{A}-\langle\hat{A}\rangle \hat{I}, \\
& |\alpha\rangle:=\Delta \hat{A}|\psi\rangle, \\
& |\beta\rangle:=\Delta \hat{B}|\psi\rangle,
\end{aligned}
$$

where $\hat{I}$ is the identity operator on $\mathscr{H}$. Then, the Schwarz inequality (4) becomes:

$$
\left\langle\psi\left|(\Delta \hat{A})^{2}\right| \psi\right\rangle\left\langle\psi\left|(\Delta \hat{B})^{2}\right| \psi\right\rangle \geq|\langle\psi|\Delta \hat{A} \Delta \hat{B}| \psi\rangle|^{2}
$$

The standard deviation of the observable $\hat{A}$ is defined as:

$$
\sigma(\hat{A}):=\left(\left\langle\psi\left|(\Delta \hat{A})^{2}\right| \psi\right\rangle\right)^{\frac{1}{2}} .
$$


Then, it is easy to see that:

$$
\begin{aligned}
\Delta \hat{A} \Delta \hat{B} & =\frac{1}{2}(\Delta \hat{A} \Delta \hat{B}+\Delta \hat{B} \Delta \hat{A})+\frac{1}{2}(\Delta \hat{A} \Delta \hat{B}-\Delta \hat{B} \Delta \hat{A}) \\
& =\frac{1}{2}\{\Delta \hat{A}, \Delta \hat{B}\}+\frac{1}{2}[\Delta \hat{A}, \Delta \hat{B}] .
\end{aligned}
$$

Since $\langle\psi|\{\Delta \hat{A}, \Delta \hat{B}\}| \psi\rangle$ is a real number and $\langle\psi|[\Delta \hat{A}, \Delta \hat{B}]| \psi\rangle$ is a pure imaginary number, the right-hand side of (9) can be rewritten as:

$$
|\langle\psi|\Delta \hat{A} \Delta \hat{B}| \psi\rangle|^{2}=\frac{1}{4}\langle\psi|\{\Delta \hat{A}, \Delta \hat{B}\}| \psi\rangle^{2}+\frac{1}{4}|\langle\psi|[\Delta \hat{A}, \Delta \hat{B}]| \psi\rangle|^{2} .
$$

Moreover, we can see that:

$$
[\Delta \hat{A}, \Delta \hat{B}]=[\hat{A}, \hat{B}]
$$

Therefore, (9) implies

$$
\begin{aligned}
\sigma(\hat{A})^{2} \sigma(\hat{B})^{2} & \geq|\langle\psi|\Delta \hat{A} \Delta \hat{B}| \psi\rangle|^{2}=\frac{1}{4}\langle\psi|\{\Delta \hat{A}, \Delta \hat{B}\}| \psi\rangle^{2}+\frac{1}{4}|\langle\psi|[\Delta \hat{A}, \Delta \hat{B}]| \psi\rangle|^{2} \\
& \geq \frac{1}{4}|\langle\psi|[\hat{A}, \hat{B}]| \psi\rangle|^{2} .
\end{aligned}
$$

By taking squre roots of the both sides, we obtain the Robertson inequality:

$$
\sigma(\hat{A}) \cdot \sigma(\hat{B}) \geq \frac{1}{2}|\langle\psi|[\hat{A}, \hat{B}]| \psi\rangle|
$$

which means that the two observables cannot have precise values simultaneously if $\langle\psi|[\hat{A}, \hat{B}]| \psi\rangle \neq 0$. On the other hand, the following quantity:

$$
\begin{aligned}
C_{s}(\hat{A}, \hat{B}) & :=\frac{1}{2}\langle\psi|\{\Delta \hat{A}, \Delta \hat{B}\}| \psi\rangle=\frac{1}{2}\langle\psi|\{\Delta \hat{A}, \hat{B}\}| \psi\rangle=\frac{1}{2}\langle\psi|\{\hat{A}, \Delta \hat{B}\}| \psi\rangle \\
& =\frac{1}{2}\langle\psi|\{\hat{A}, \hat{B}\}| \psi\rangle-\langle\psi|\hat{A}| \psi\rangle\langle\psi|\hat{B}| \psi\rangle,
\end{aligned}
$$

is called the symmetrized covariance of $\hat{A}$ and $\hat{B}$. Then, (14) can be rewritten as:

$$
\sigma(\hat{A})^{2} \cdot \sigma(\hat{B})^{2} \geq\left|C_{s}(\hat{A}, \hat{B})\right|^{2}+\frac{1}{4}|\langle\psi|[\hat{A}, \hat{B}]| \psi\rangle|^{2} .
$$

Sometimes this is referred to as the Schrödinger inequality [10].

\section{Angular order parameter and orbital angular momentum}

In this section, we show our main result. Let us consider a quantum mechanical particle in a configuration space whose dimensionality is equal to or larger than two. The system has four observables $\hat{x}, \hat{y}, \hat{p}_{x}, \hat{p}_{y}$, which satisfy the canonical commutation relations $\left[\hat{x}_{j}, \hat{p}_{k}\right]=i \hbar \delta_{j k}$. We introduce two operators:

$$
\hat{Z}:=\hat{x}+i \hat{y}, \quad \hat{L}:=\hat{x} \hat{p}_{y}-\hat{y} \hat{p}_{x}
$$

The operator $\hat{Z}$ is not self-adjoint but it is related to position of the particle. The self-adjoint operator $\hat{L}$ is called the orbital angular momentum (OAM). They satisfy the following:

$$
[\hat{L}, \hat{Z}]=\hbar \hat{Z}
$$

and:

$$
\left[\hat{L}, \hat{Z}^{n}\right]=n \hbar \hat{Z}^{n}
$$


for any natural number $n=1,2,3, \ldots$ With a normalized vector $\psi \in \mathscr{H}$ we define:

$$
\langle\hat{L}\rangle:=\langle\psi|\hat{L}| \psi\rangle, \quad \Delta \hat{L}:=\hat{L}-\langle\hat{L}\rangle \hat{I} .
$$

By substituting:

$$
|\alpha\rangle=\Delta \hat{L}|\psi\rangle, \quad|\beta\rangle=\hat{Z}|\psi\rangle,
$$

into the Schwarz inequality (4) and by noting $\langle\alpha|=\langle\psi| \Delta \hat{L}^{\dagger}=\langle\psi| \Delta \hat{L}$ and $\langle\beta|=\langle\psi| \hat{Z}^{\dagger}$, we get:

$$
\left\langle\psi\left|(\Delta \hat{L})^{2}\right| \psi\right\rangle\left\langle\psi\left|\hat{Z}^{\dagger} \hat{Z}\right| \psi\right\rangle \geq|\langle\psi|\Delta \hat{L} \hat{Z}| \psi\rangle|^{2} .
$$

Hence:

$$
\sqrt{\left\langle(\Delta \hat{L})^{2}\right\rangle} \sqrt{\left\langle\hat{Z}^{\dagger} \hat{Z}\right\rangle} \geq|\langle\Delta \hat{L} \hat{Z}\rangle| .
$$

In a similar way, by substituting:

$$
|\alpha\rangle=\hat{Z}^{\dagger}|\psi\rangle, \quad|\beta\rangle=\Delta \hat{L}|\psi\rangle,
$$

into (4), we get:

$$
\sqrt{\left\langle\hat{Z} \hat{Z}^{\dagger}\right\rangle} \sqrt{\left\langle(\Delta \hat{L})^{2}\right\rangle} \geq|\langle\hat{Z} \Delta \hat{L}\rangle| .
$$

Note that $\hat{Z} \hat{Z}^{\dagger}=\hat{Z}^{\dagger} \hat{Z}$. The triangle inequality $|a|+|b| \geq|a-b|$ holds for any complex number $a, b$. The commutation relation (19) implies $[\Delta \hat{L}, \hat{Z}]=[\hat{L}, \hat{Z}]=\hbar \hat{Z}$. By adding (24) with (26) and multiplying $1 / 2$, we obtain:

$$
\begin{aligned}
\sqrt{\left\langle(\Delta \hat{L})^{2}\right\rangle} \sqrt{\left\langle\hat{Z}^{\dagger} \hat{Z}\right\rangle} & \geq \frac{1}{2}\{|\langle\Delta \hat{L} \hat{Z}\rangle|+|\langle\hat{Z} \Delta \hat{L}\rangle|\} \\
& \geq \frac{1}{2}\{|\langle\Delta \hat{L} \hat{Z}-\hat{Z} \Delta \hat{L}\rangle|\} \\
& =\frac{1}{2} \hbar|\langle\hat{Z}\rangle| .
\end{aligned}
$$

This is one of our main results.

By replacing the operator $\hat{Z}$ with $\hat{Z}^{n}$, we can derive more general inequalities:

$$
\sqrt{\left\langle(\Delta \hat{L})^{2}\right\rangle} \sqrt{\left\langle\left(\hat{Z}^{\dagger} \hat{Z}\right)^{n}\right\rangle} \geq \frac{1}{2} n \hbar\left|\left\langle\hat{Z}^{n}\right\rangle\right| \quad(n=1,2,3, \ldots),
$$

via a similar inference. The nonnegative number:

$$
\sigma(\hat{L}):=\sqrt{\left\langle\psi\left|(\Delta \hat{L})^{2}\right| \psi\right\rangle}=\left\langle\psi\left|\hat{L}^{2}\right| \psi\right\rangle-\langle\psi|\hat{L}| \psi\rangle^{2},
$$

is the standard deviation of the orbital angular momentum. The complex number

$$
\left\langle\hat{Z}^{n}\right\rangle=\left\langle\psi\left|(\hat{x}+i \hat{y})^{n}\right| \psi\right\rangle=\iint_{-\infty}^{\infty}(x+i y)^{n}|\psi(x, y)|^{2} d x d y
$$

is the $n$-th moment of probability density for the wave function $\psi(x, y)^{1}$. If the probability density $|\psi(x, y)|^{2}$ is rotationally invariant, all the moments vanish $\left\langle\hat{Z}^{n}\right\rangle=0(n=1,2,3, \ldots)$. Conversely, if the system exhibits a nonvanishing moment $\left\langle\hat{Z}^{n}\right\rangle \neq 0$ for some $n$, the probability density, $|\psi(x, y)|^{2}$, is not rotationally invariant. Hence, the expectation value

\footnotetext{
${ }^{1}$ If the dimension of the configuration space is larger than two, it is necessary to use a suitable wave function $\psi(x, y, x, \ldots)$.
} 
$\left\langle\hat{Z}^{n}\right\rangle$ is interpreted as an order parameter to measure the degree of breaking of the rotational symmetry. The complex number:

$$
\mu_{n}:=\frac{\left\langle\hat{Z}^{n}\right\rangle}{\sqrt{\left\langle\left(\hat{Z}^{\dagger} \hat{Z}\right)^{n}\right\rangle}}=\frac{\left\langle(\hat{x}+i \hat{y})^{n}\right\rangle}{\sqrt{\left\langle\left(\hat{x}^{2}+\hat{y}^{2}\right)^{n}\right\rangle}},
$$

is called the normalized n-th moment of position distribution or the normalized angular order parameter, which indicates bias or asymmetry of angular distribution of the particle. Then, we have:

$$
\sigma(\hat{L}) \geq \frac{1}{2} n \hbar \frac{\left|\left\langle\hat{Z}^{n}\right\rangle\right|}{\left\langle\left(\hat{Z}^{\dagger} \hat{Z}\right)^{n}\right\rangle^{1 / 2}}=\frac{1}{2} n \hbar\left|\mu_{n}\right| \quad(n=1,2,3, \ldots) .
$$

This is the main result of our work. This inequality implies that if the uncertainty $\sigma(\hat{L})$ of OAM is small, the normalized moment $\left|\mu_{n}\right|$ must be small. In this case, the angular distribution is not strongly biased and hence the uncertainty of angle must be large.

However, if the uncertainty of angle is small, the angular distribution is strongly biased and hence, the normalized moment $\left|\mu_{n}\right|$ becomes large, then the inequality (32) implies that the uncertainty $\sigma(\hat{L})$ of OAM must become large.

\section{Tighter inequality}

The necessary and sufficient conditions for the equality in (27) are the two equalities in (24), (26) and the other equality $\langle\Delta \hat{L} \hat{Z}\rangle=-\langle\hat{Z} \Delta \hat{L}\rangle$. Actually, there is no state vector satisfying these three conditions simultaneously, and hence, the equality in (27) is never attained. In this sense, the inequality (27) is not tight. operators:

It is desirable to find a tighter inequality. For this purpose, we introduce self-adjoint

$$
\hat{x}_{n}:=\frac{1}{2}\left(\hat{Z}^{n}+\hat{Z}^{\dagger n}\right) \quad \hat{y}_{n}:=\frac{1}{2 i}\left(\hat{Z}^{n}-\hat{Z}^{\dagger n}\right),
$$

for $n=1,2,3, \ldots$ Then, we have:

$$
\hat{Z}^{n}=(\hat{x}+i \hat{y})^{n}=\hat{x}_{n}+i \hat{y}_{n} .
$$

Using these, it is easy to see that:

$$
\begin{aligned}
\Delta \hat{L} \hat{Z}^{n} & =\frac{1}{2}\left\{\Delta \hat{L}, \hat{Z}^{n}\right\}+\frac{1}{2}\left[\Delta \hat{L}, \hat{Z}^{n}\right] \\
& =\frac{1}{2}\left\{\Delta \hat{L},\left(\hat{x}_{n}+i \hat{y}_{n}\right)\right\}+\frac{1}{2} n \hbar \hat{Z}^{n} \\
& =\frac{1}{2}\left\{\Delta \hat{L}, \hat{x}_{n}\right\}+i \frac{1}{2}\left\{\Delta \hat{L}, \hat{y}_{n}\right\}+\frac{1}{2} n \hbar\left(\hat{x}_{n}+i \hat{y}_{n}\right) .
\end{aligned}
$$

Hence, (23) is equivalent to:

$$
\begin{aligned}
\left\langle(\Delta \hat{L})^{2}\right\rangle \cdot\left\langle\hat{Z}^{\dagger} \hat{Z}\right\rangle & \geq\left|\frac{1}{2}\left\langle\left\{\Delta \hat{L}, \hat{x}_{n}\right\}\right\rangle+\frac{1}{2} n \hbar\left\langle\hat{x}_{n}\right\rangle\right|^{2}+\left|\frac{1}{2}\left\langle\left\{\Delta \hat{L}, \hat{y}_{n}\right\}\right\rangle+\frac{1}{2} n \hbar\left\langle\hat{y}_{n}\right\rangle\right|^{2} \\
& =\left|C_{s}\left(\hat{L}, \hat{x}_{n}\right)+\frac{1}{2} n \hbar\left\langle\hat{x}_{n}\right\rangle\right|^{2}+\left|C_{s}\left(\hat{L}, \hat{y}_{n}\right)+\frac{1}{2} n \hbar\left\langle\hat{y}_{n}\right\rangle\right|^{2} .
\end{aligned}
$$

This is the tightest inequality whose equality can be attained. However, the equality holds if and only if the state is an eigenstate of $\hat{L}$. In this case, both sides of (36) are zero. 


\section{Experimental realization}

We have formulated the uncertainty relations (27), (32), (36). In order to test these relations, we need to have a method for controlling and measuring angular momenta of particles.

In optics, there is a method for controlling and measuring angular momenta of photons. Franke-Arnold and Padgett et al. [11,12] have tested the uncertainty relation of Judge [4] and Berbett, Pegg [7], by using an analyzer of photon angular momentum.

Uchida and Tonomura [13] first made a coherent electron beam carrying nonzero orbital angular momentum. Such electron beam has a wave front whose shape looks like a vortex. Verbeeck et al. [14] and McMorran et al. [15] developed fork-shaped diffraction gratings, which control orbital angular momenta of electrons. They observed circularly symmetric diffraction patterns for eigenstates of orbital angular momentum. Thus, they verified that the uncertainty in angular distribution was maximum when the uncertainty of angular momentum was minimum.

Recently, Hasegawa and Saitoh et al. [16,17] made a superposition of two coherent electron beams carrying different angular momenta. As a result, they produced a quantum state that has an uncertain orbital angular momentum. They observed an interference pattern that was circularly asymmetric. Thus, they verified that the uncertainty in angular distribution became smaller when the uncertainty of angular momentum became larger. Yet, quantitative analysis of the uncertainty relation was not performed in experiments using electrons.

\section{Generalization}

The angular momentum $\hat{L}$ is a generator of rotational transformations, which transform the angle variable $(\hat{x}+i \hat{y}) / \sqrt{\hat{x}^{2}+\hat{y}^{2}}$. A nonzero value of the order parameter $\mu=\langle\hat{x}+i \hat{y}\rangle / \sqrt{\left\langle\hat{x}^{2}+\hat{y}^{2}\right\rangle}$ indicates breaking of rotational symmetry, or certainty of the angle distribution, which accompanies uncertainty of the angular momentum. The relation between the angle order parameter and the uncertainty of the angular momentum is expressed by the inequality (32).

This kind of relation between a symmetry generator and a symmetry-breaking order parameter can be formulated in a more general form. Suppose that we have a self-adjoint operator $\hat{G}$, which is a generator of symmetry transformations and is called charge. Also, suppose that we have another operator, $\hat{\Phi}$. It is not necessary to assume that $\hat{\Phi}$ is a self-adjoint operator. Then, the inequality:

$$
\sigma(\hat{G}) \geq \frac{|\langle[\hat{G}, \hat{\Phi}]\rangle|}{\sqrt{\left\langle\hat{\Phi}^{\dagger} \hat{\Phi}\right\rangle}+\sqrt{\left\langle\hat{\Phi} \hat{\Phi}^{\dagger}\right\rangle}},
$$

holds. The expectation value $\langle[\hat{G}, \hat{\Phi}]\rangle=\langle\psi|[\hat{G}, \hat{\Phi}]| \psi\rangle$ is taken with respect to a state $|\psi\rangle$. This is a generalization of (27) and its proof is straightforward.

On the left-hand side of (37), the standard deviation $\sigma(\hat{G})$ measures uncertainty of the charge, while on the right-hand side of (37), the commutator $[\hat{G}, \hat{\Phi}]$ represents transformation of $\hat{\Phi}$ by $\hat{G}$. If the state $|\psi\rangle$ is invariant under the transformation generated by $\hat{G}$, then $\langle\psi|[\hat{G}, \hat{\Phi}]| \psi\rangle=0$. If the order parameter $\langle[\hat{G}, \hat{\Phi}]\rangle$ exhibits a nonzero value, then the state is not invariant and the uncertainty of the charge must satisfy inequality (37). 
This formulation is applicable to the uncertainty relation between the particle number and the phase. In this case, we take $\hat{G}=\hat{a}^{\dagger} \hat{a}$ and $\hat{\Phi}=\hat{a}$, with the creation and annihilation operators $\hat{a}^{\dagger}$ and $\hat{a}$.

This formulation is applicable also to the complementarity relation [18] between the particle and wave natures.

\section{Summary}

The uncertainty relation between angle and orbital angular momentum does not have a formulation similar to the uncertainty relation between position and linear momentum. The angle variable is not represented by a quantum mechanical self-adjoint operator, although the other observables are represented by self-adjoint operators. We reviewed the general formulation of the uncertainty relation between noncommutative observables, which was proved by Robertson. Instead of the angle variable operator, we introduced the complex position operator $\hat{Z}=\hat{x}+i \hat{y}$ and interpreted the order parameter $\mu=\langle\hat{Z}\rangle / \sqrt{\left\langle\hat{Z}^{\dagger} \hat{Z}\right\rangle}$ as a measure of certainty of angle distribution. Then, we have proven relation (27) between the uncertainty of angular momentum and the certainty of angle. We proved relations (32), which are generalizations to higher moments of angular distribution $\mu_{n}=\left\langle\hat{Z}^{n}\right\rangle / \sqrt{\left\langle\left(\hat{Z}^{\dagger} \hat{Z}\right)^{n}\right\rangle}$. We proved also the tightest inequality (36). A theoretical generalization to the uncertainty relation (37) between a symmetry generator and an order parameter was shown. Methods for controlling angular momenta of photons and electrons were discussed. Quantitative experimental tests of the relations will be discussed in future publications.

In this paper, we considered uncertainties of values of observables that are inherent in quantum states. However, we did not consider measurement process of observables. An actual measurement process involves measurement error and causes disturbance on the state of the measured system. Ozawa [19] formulated a quantitative relation between the measurement error and the disturbance. Branciard [20] established the tightest inequality that the error and the disturbance obey. We do not yet know this kind of error-disturbance relation for the angle and angular momentum.

Hayashi [21] formulated quantum estimation theory for the group action, which can be regarded as a generalization of the problem that was considered in our work. This aspect warrants further investigation.

\section{Acknowledgements}

The author thanks Keisuke Watanabe, who discussed with me the tighter version of the uncertainty inequality, Eq. (36). He thanks Prof. Katsuhiro Nakamura and Prof. Davron Matrasulov for their warm hospitality for supporting his stays in Uzbekistan. This manuscript is written as a part of the proceedings of the workshop, Wave dynamics in low-dimensional branched structures, held during September 23-24, 2014 in Tashkent, Uzbekistan. This work is financially supported by the Grant-in-Aid for Scientific Research of Japan Society for the Promotion of Science, Grant No. 26400417.

\section{References}

[1] Heisenberg W. Über den anschaulichen Inhalt der quantentheoretischen Kinematik und Mechanik. $Z$. Physik, 43, P. 172 (1927).

[2] Robertson H.P. The uncertainty principle. Phys. Rev., 34, P. 163 (1929).

[3] Schiff L.I. Quantum Mechanics, 3rd edition, McGraw-Hill (1968).

[4] Judge D. On the uncertainty relation for $L_{z}$ and $\varphi$. Phys. Lett., 5, P. 189 (1963). 
[5] Kraus K. Remark on the uncertainty between angle and angular momentum. Z. Physik, 188, P. 374 (1965).

[6] Carruthers P., Nieto M.M. Phase and angle variables in quantum mechanics. Rev. Mod. Phys., 40, P. 411 (1968).

[7] Bernett S.M., Pegg D.T. Quantum theory of rotation angles. Phys. Rev. A, 41, P. 3427 (1990).

[8] Ohnuki Y., Kitakado S. Fundamental algebra for quantum mechanics on $S^{D}$ and gauge potentials. $J$. Math. Phys., 34, P. 2827 (1993).

[9] Tanimura S. Gauge field, parity and uncertainty relation of quantum mechanics on $S^{1}$. Prog. Theor. Phys., 90, P. 271 (1993).

[10] Schrödinger E. Zum Heisenbergschen Unschärfeprinzip. Sitzungsberichte der Preussischen Akademie der Wissenschaften, Physikalisch-mathematische Klasse, 19, P. 296 (1930).

[11] Franke-Arnold S., Barnett S.M., et al. Uncertainty principle for angular position and angular momentum. New J. Phys., 6, P. 103 (2004).

[12] Pegg D.T., Barnett S.M., et al. Minimum uncertainty states of angular momentum and angular position. New J. Phys., 7, P. 62 (2005).

[13] Uchida M. Tonomura A. Generation of electron beams carrying orbital angular momentum. Nature, 464, P. 737 (2010).

[14] Verbeeck J., Tian H., Schattschneider P. Production and application of electron vortex beams. Nature, 467, P. 301 (2010).

[15] McMorran B.J., Agrawal A., et al. Electron vortex beams with high quanta of orbital angular momentum. Science, 331, P. 192 (2011).

[16] Hasegawa Y., Saitoh K., et al. Young's interference experiment with electron beams carrying orbital angular momentum. J. Phys. Soc. Jap., 82, P. 033002 (2013).

[17] Hasegawa Y., Saitoh K., Tanaka N., Uchida M. Propagation dynamics of electron vortex pairs. J. Phys. Soc. Jap., 82, P. 073402 (2013).

[18] Tanimura S. The incompatibility relation between visibility of interference and distinguishability of paths. ArXiv quant-ph/0703118 (2007).

[19] Ozawa M. Universally valid reformulation of the Heisenberg uncertainty principle on noise and disturbance in measurement. Phys. Rev. A, 67, P. 042105 (2003).

[20] Branciard C. Error-tradeoff and error-disturbance relations for incompatible quantum measurements. Proceedings of the National Academy of Science of the USA, 110 (17), P. 6742 (2013).

[21] Hayashi $M$. Fourier analytic approach to quantum estimation of group action. ArXiv 1209.3463v2 (2012). 